Carbohydrate Research, 84 (1980) 273-285

Elsevier Scientific Publishing Company, Amsterdam - Printed in The Netherlands

\title{
STRUCTURE OF THE DEXTRAN OF Leuconostoc mesenteroides B-1355*
}

\author{
AKIRA Misaki ${ }^{\dagger}$, \\ Faculty of Science of Living, Osaka City University, Sugimoro-cho, Sumiyoshi, Osaka 558 (Japan) \\ MITSUO TORI, \\ Institute for Microbial Diseases, Osaka University, Yamadakami, Suita, Osaka 565 (Japan) \\ Teruo SawaI, \\ Department of Biology, Aichi Kyoiku University, Kariya, Aichi 448 (Japan) \\ AND IRWIN J. GOLDSTEN \\ Department of Biological Chemistry, Medical School, The University of Michigan, Ann Arbor, Michigan \\ 48109 (U.S.A.)
}

(Received February 3rd, 1978; accepted for publication in revised form, January 15th, 1980)

\section{ABSTRACT}

The structure of dextran B-1355-S (soluble fraction) has been investigated. Acid hydrolysis of the methylated dextran yielded 2,3,4,6-tetra-, 2,3,4-tri-, 2,4,6-tri-, and 2,4-di- $O$-methyl-D-glucose, in the molar ratios of $1.0: 4.6: 3.6: 1.0$, indicating that the dextran has a branched structure containing $(1 \rightarrow 6)$ - and $(1 \rightarrow 3)-\alpha-D$-glucosidic linkages with an average repeating-unit of ten sugar residues. The dextran-polyalcohol, derived by successive periodate oxidation and borohydride reduction, gave, on complete hydrolysis with acid, glycerol and glucose (molar ratio, 1.0:0.83); mild hydrolysis $\left(0.1 \mathrm{M}\right.$ acid at $\left.25^{\circ}\right)$ gave glycerol and $1-O-\alpha-\mathrm{D}$-glucosylglycerol (molar ratio, $1: 1.45$ ).

Exo- $\mathrm{G}_{2}$-dextranase of Arthrobacter globiformis $\mathrm{T} 6$ acted on the dextran to release isomaltose and $O$ - $\alpha$-D-glucopyranosyl-( $(\rightarrow 3)-O-\alpha$-D-glucopyranosyl-( $(1 \rightarrow 6)-$ D-glucose in the molar ratio of 5.7:1.0, leaving a degraded dextran (limit dextran). Methylation analysis indicated the limit-dextran to be highly branched, with a repeating unit of six glucose residues, consisting mainly of alternate $(1 \rightarrow 6)$ - and $(1 \rightarrow 3)-\alpha$-D-glucosidic linkages.

These findings confirm the overall arrangement of alternating $(1 \rightarrow 6)$ - and $(1 \rightarrow 3)$-linked $\alpha$-D-glucose residues, and the absence of consecutive $\alpha$ - $(1 \rightarrow 3)$-linked sugar residues; the latter factor may be related to the water insolubility of $\alpha$-D-glucans of Streptococcus mutans.

\footnotetext{
*A part of this work was presented at the Annual Meeting of the Japanese Biochemical Society, Fukuoka, October 13-16, 1975, and at the 8th International Symposium on Carbohydrate Chemistry, Kyoto, August 16-18, 1976.

tTo whom reprint requests should be sent.
} 
INTRODUCTION

Most dextrans elaborated by Leuconostoc mesenteroides and related microorganisms contain a substantial number of $(1 \rightarrow 6)-\alpha$-D-glucosidic bonds, together with various proportions of $(1 \rightarrow 2)-,(1 \rightarrow 3)-$, and $(1 \rightarrow 4)-\alpha-D$-glucosidic linkages (see the extensive, recent review by Sidebotham ${ }^{1}$ ). These non- $(1 \rightarrow 6)$ linkages usually occur as the origin of branch points, as in the case of dextran ${ }^{2}$ B-512 and dextran 3,4 B-1397. However, certain dextrans, such as the difficulty water-soluble fractions of dextran B-1299, have been postulated to contain consecutive $(1 \rightarrow 3)-\alpha$-D-glucosidic linkages ${ }^{5,6}$. Considerable attention has recently focused on the physical properties of water-insoluble $\alpha$-D-glucans of cariogenic oral streptococci. Our recent study established that the insoluble glucan-fraction of the $\alpha$-D-glucan of Streptococcus mutans OMZ 176 consists of an $\alpha-(1 \rightarrow 3)$-linked backbone chain, carrying short $(1 \rightarrow 6)$-linked side-chains ${ }^{7}$.

During investigations on the relationship between the structure and the physical and immunochemical properties of dextrans and related $\alpha$-D-glucans, we became interested in the water-soluble fraction of dextran B-1355 (dextran B-1355-S), which was previously reported to contain a high proportion of $(1 \rightarrow 3)$-linked $D$-glucose residues, namely, $35 \%$ as shown by a periodate-oxidation study ${ }^{8}$, and $47 \%$ by Smith degradation ${ }^{9}$. In contrast to the insoluble glucans of Streptococcus mutans, however, dextran B-1355-S is readily soluble in water. From the acetolysis product of this dextran, Goldstein and Whelan isolated nigerose (20\%) and 3-O-isomaltosylD-glucose $(2 \%)$, in addition to glucose and isomaltose, and suggested the presence of alternate $\alpha-(1 \rightarrow 3)$ and $(1 \rightarrow 6)$ linkages in the same molecule ${ }^{10}$.

This paper reports the detailed structural features of dextran B-1355-S with special reference to the sequences of $(1 \rightarrow 3)$ - and $(1 \rightarrow 6)$-linked $\alpha$-D-glucose residues, as revealed by cherical investigations and enzymic-degradation studies.

A recent paper by Seymour et al. ${ }^{11}$ described the methylation data for dextran B-1355-S as well as several other dextrans.

RESULTS AND DISCUSSION

Dextran B-1355-S $\left([\alpha]_{D}+220^{\circ}\right)$ was methylated twice by the method of Hakomori ${ }^{12}$. The permethylated dextran was hydrolyzed with acid, and the methylated-sugar fragments were analyzed by g.l.c.-m.s. as their alditol acetates, and also as their methyl glycosides. The identities and molar ratios of the cleavage fragments are listed in Table I, showing the presence of 2,3,4,6-tetra-, 2,3,4-tri-, 2,4,6-tri-, and 2,4-di- $O$-methyl-D-glucose, in the molar ratios of 1.0:4.6:3.6:1.0. Although these data are very close to those obtained by Seymour et al. ${ }^{11}$, our results are based on a more-completely methylated dextran, compared with the previous data which appeared to involve a slightly under-methylated product. Our results indicate that dextran B-1355-S possesses a branched structure having an average repeating-unit of approximately ten glucose residues, of which 3 or 4 glucose residues are joined by 
TABLE I

MOLAR RATIO OF METHYLATED D-GLUCOSE FRAGMENTS FROM HYDROLYZATES OF METHYLATED DEXTRAN B-1355-S, DEXTRAN-POLYALCOHOL, AND G2-DEXTRANASE LIMIT-DEXTRIN

\begin{tabular}{|c|c|c|c|}
\hline O-Methyl-D-glucose & $\begin{array}{l}\text { Native } \\
\text { dextran }\end{array}$ & $\begin{array}{l}\text { Dextran- } \\
\text { polyalcohol }\end{array}$ & $\begin{array}{l}G_{2} \text {-Dextranase } \\
\text { limit-dextran }\end{array}$ \\
\hline 2,3,4,6-Tetra- & 1.0 & (trace) & 1.0 \\
\hline 2,3,4-Tri- & 4.6 & & 2.4 \\
\hline 2,4,6-Tri- & 3.6 & 3.64 & 1.47 \\
\hline 2,4-Di- & 1.0 & 1.0 & 1.0 \\
\hline
\end{tabular}

$\alpha-(1 \rightarrow 3)$, and 4 or 5 other residues are joined by $\alpha-(1 \rightarrow 6)$ linkages, respectively; branching occurs by substitution at $\mathrm{O}-3$ and $\mathrm{O}-6$ of the $\mathrm{D}$-glucosyl residues.

The methylation data suggest that the gross structure of dextran B-1355-S resembles that of the insoluble glucan of Streptococcus mutans OMZ 176, which also has a branched structure (average repeating-unit, seven sugar residues) consisting of $(1 \rightarrow 3)$ - and $(1 \rightarrow 6)-\alpha$-D-glucosidic linkages in the ratio ${ }^{7}$ of $5: 2$.

In order to obtain further information on the structure of dextran B-1355-S, the polysaccharide was oxidized with periodate at $20^{\circ}$. After complete oxidation ( 7 days; periodate consumption $1.15 \mathrm{~mol}$, and formic acid production $0.61 \mathrm{~mol}$ per glucose residue), the oxidized dextran was reduced with sodium borohydride to afford the dextran-polyalcohol. Methylation of the dextran-polyalcohol followed by acid hydrolysis yielded 2,4,6-tri- and 2,4-di- $O$-methylglucose in the molar ratio of 3.64:1.0, and a trace of 2,3,4,6-tetra- $O$-methylglucose (Table I). This result confirms that all $(1 \rightarrow 6)$-linked $D$-glucose residues and non-reducing terminal groups were completely cleaved by periodate. When the dextran-polyalcohol was hydrolyzed by heating it with $0.25 \mathrm{M}$ sulfuric acid (complete Smith-degradation) ${ }^{13}$, glycerol and glucose were formed in the molar ratio of 1.0:0.83, as estimated by g.l.c. of the acetylated polyalcohols. This result is in good agreement with the methylation data.

Unambiguous evidence for alternating $(1 \rightarrow 3)$ - and $(1 \rightarrow 6)-\alpha-D$-glucosidic linkages was provided by examination of the products from mild acid hydrolysis of the dextran-polyalcohol (mild Smith-degradation) ${ }^{14}$. When the polyalcohol was hydrolyzed with $0.05 \mathrm{M}$ sulfuric acid at $25^{\circ}$, glycerol and 1-O- $\alpha$-D-glucosylglycerol $\left([\alpha]_{\mathrm{D}}+134.8^{\circ}\right)$ were produced, the latter glucoside being identified as the crystalline acetate, and also by g.l.c. as its $O$-trimethylsilyl derivative. The molar ratio of 1-O$\alpha$-D-glucosylglycerol to glycerol was estimated to be $1.45: 1.0$. As the glucosylglycerol was derived from the sequence $\rightarrow 6)-\alpha-D-G l c p-(1 \rightarrow 3)-\alpha-D-G l c p-(1 \rightarrow 6)-D-G l c p-(1 \rightarrow$, there must be a considerable proportion of alternating $\alpha-(1 \rightarrow 3)$ and $(1 \rightarrow 6)$ linkages. The fact that the glucosylglycerol was the major product, and higher oligosaccharides (such as glycerol glucosides of $\alpha-(1 \rightarrow 3)$-linked nigero-oligosaccharides) were not detectable by paper-chromatographic examination, strongly suggests that dextran 


\section{TABLE II}

EFFECTS OF $\alpha$-D-GLUCAN HYDROLASES ON DEXTRAN B-1355-S AND OMZ 176 MUTAN

Enzyme $\quad$ pH Hydrolysis Percentage ${ }^{a}$

Dextran B-1355-S OMZ 176 Mutan

\begin{tabular}{llrc}
\hline Penicillum sp. dextranase & 6 & $7.3^{b}$ & $11.0^{b}$ \\
G2-Dextranase & 4.5 & $22.2^{c}$ & $5.0^{c}$ \\
Exo-(1-6)-D-glucosidase & 5.5 & $3.5^{b}$ & $11.3^{b}$ \\
$(1 \rightarrow 3)-\alpha-\nu-$ Glucan hydiolase & 6.3 & $0.5^{b}$ & 11.3
\end{tabular}

${ }^{a}$ Determined by reducing value after incubation for $24 \mathrm{~h}$ at $37^{\circ},{ }^{b}$ Expressed as glucose. ${ }^{c}$ Expressed as isomaltose.

B-1355-S, unlike the insoluble glucan of Streptococcus mutans OMZ176, consists mainly of alternating $(1 \rightarrow 3)$ - and $(1 \rightarrow 6)$ - $\alpha-D$-glucosidic linkages.

The action of various $\alpha$-D-glucan hydrolases on dextran B-1355-S and the glucan of Streptococcus mutans OMZ 176 (abbreviated as OMZ 176 mutan) was compared. As shown in Table II, a dextranase preparation of Penicillum sp. (commercial product) hydrolyzed both polysaccharides to similar extents, with release of glucose, isomaltose, and other oligosaccharides. ( $1 \rightarrow 3)-\alpha-D-G l u c a n$ hydrolase (EK-14 enzyme), purified from the culture fluid of Flavobacterium sp. ${ }^{15}$, hydrolyzed OMZ 176 mutan, with the release of nigerose and several nigerosaccharides, as reported previously ${ }^{15}$, but it failed to attack dextran B-1355-S. On the other hand, the isomalto $\left(\mathrm{G}_{2}\right)$-dextranase of Arthrobacter globiformis T6 (ref. 16), which hydrolyzes $(1 \rightarrow 6)-\alpha$-D-glucosidic linkages in dextrans from the nonreducing termini with the release of isomaltose groups ${ }^{17}$, scarcely acted on OMZ i76 mutan, whereas it hydrolyzed dextran B-1355-S to a marked extent (apparent hydrolysis, 38.2\%). However, when dextran B-1355-S was incubated with an exo-(1 $\rightarrow 6)-\alpha-D$-glucosidase of Arthrobacter globiformis ${ }^{18}$, only $3.5 \%$ of glucose was liberated. These results clearly indicate an essential difference between dextran B-1355-S and OMZ 176 mutan in the arrangements of D-glucosidic linkages.

For characterization of the products obtained from the $\mathrm{G}_{2}$-dextranase digestion of dextran B-1355-S, the enzyme digest was subjected to gel filtration on a column of Sephadex G-25, whereby two distinct fractions, one of high molecular weight (F-1) and one of low molecular weight (F-2) were obtained (Fig. 1). Paper chromatography (6:1:3, 1-propanol-acetic acid-water) revealed that Fraction 2 contained only isomaltose and a trisaccharide having $R_{\text {isomaltose }} 0.83$. The trisaccharide, which showed $[\alpha]_{\mathrm{D}}+130^{\circ}$, was separated from isomaltose on a thick filter-paper sheet. Methylation and acid-hydrolysis of the purified trisaccharide gave equal proportions of 2,3,4,6-tetra-, 2,4,6-tri-, and 2,3,4-tri- $O$-methyl-D-glucose, as identified by g.l.c. of their alditol acetates. Incubation of the trisaccharide with yeast $\alpha$-D-glucosidase gave glucose and isomaltose, as detected by paper chromatography. From the 


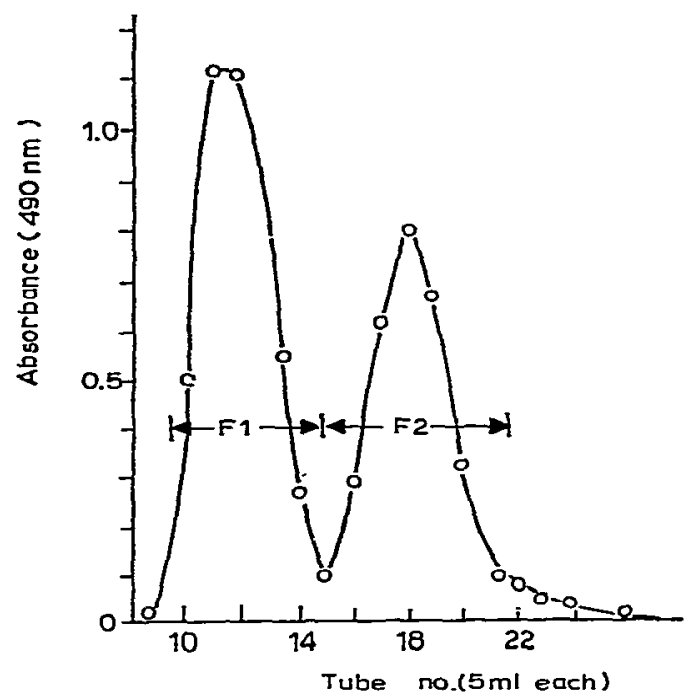

Fig. 1. Gel-filtration profile of the $\mathrm{G}_{2}$-dextranase digest of dextran B-1355-S on Sephadex G-25.

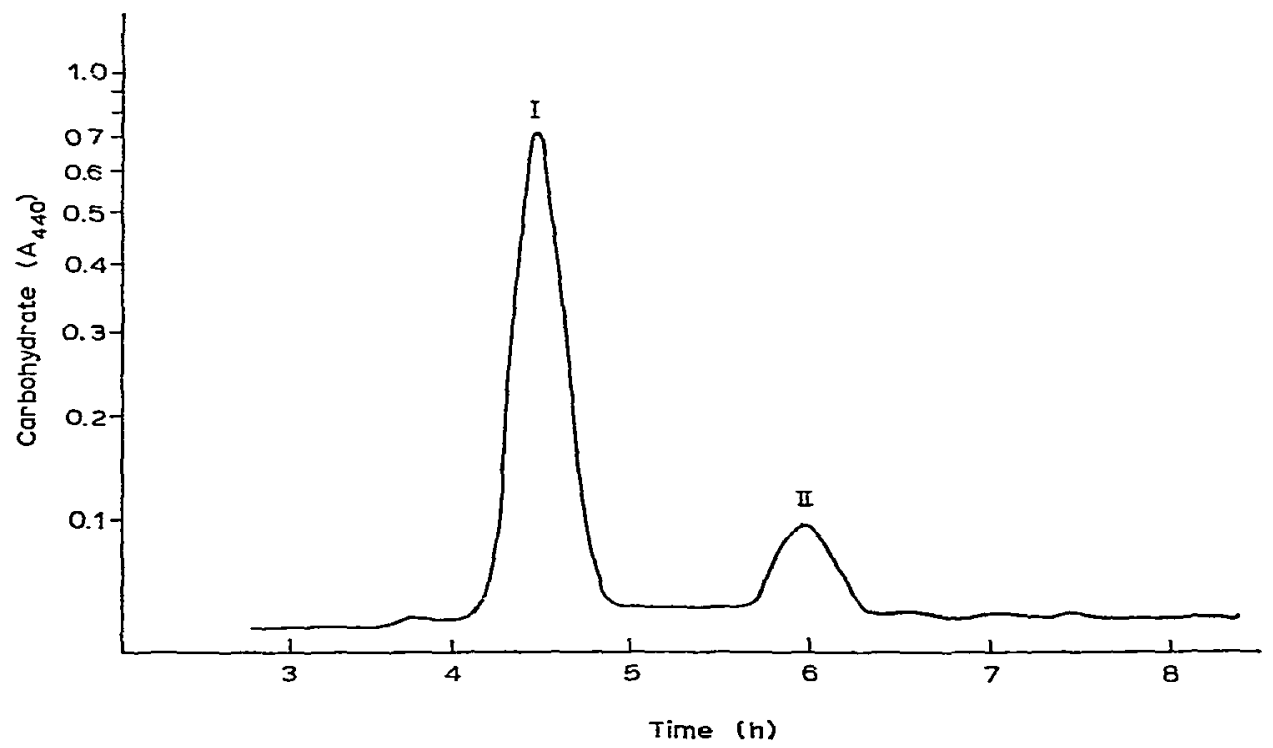

Fig. 2. Liquid-chromatographic separation of oligosaccharides obtained by $\mathrm{G}_{2}$-dextranase digestion of dextran B-1355-S. I, Isomaltose; II, 3-O- $\alpha$-D-glucosylisomaltose. An arrow indicates the position of 6-O- $\alpha$-D-glucosylnigerose.

methylation and enzymic-degradation data, the trisaccharide was identified as $O-\alpha$-D-glucosyl-( $1 \rightarrow 3)-O-\alpha$-D-glucosyl-(1 $\rightarrow 6$ )-D-glucose (or 3-O- $\alpha$-D-glucosylisomaltose). The isomaltose and the aforementioned trisaccharide in Fraction 2 were also analyzed by automated liquid-chromatography with a column of anion-exchange 


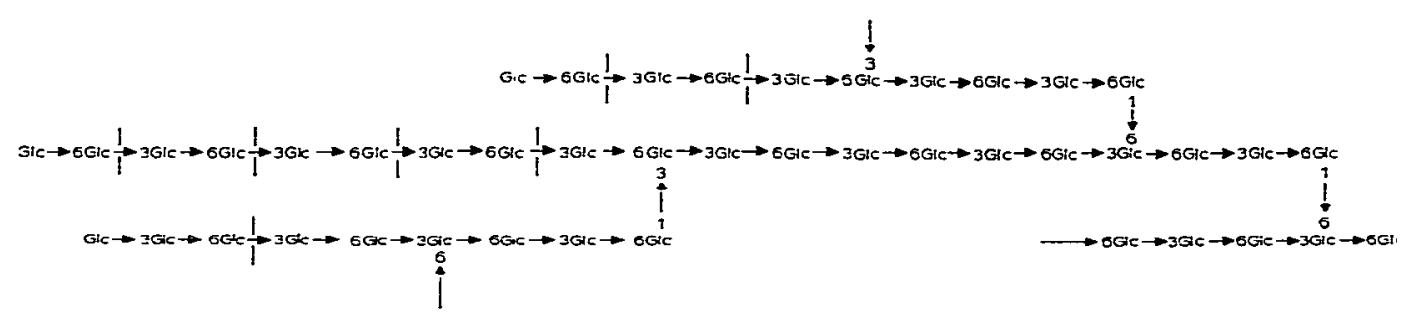

Fig. 3. Ramified structural model of dextran 1355-S, and the hydrolytic cleavages of external chains by $\mathrm{G}_{2}$-dextranase. Glc: $\alpha$-D-glucopyranose residue: |' cleavage point by the action of enzyme.

resin (Jeol LCR-3) and stepwise elution with borate buffers ${ }^{19}$. The first peak (Fig. 2) corresponds to isomaltose and the second to 3-O- $\alpha$-D-glucosylisomaltose, and not to 6-O- $\alpha-\mathrm{D}$-glucosylnigerose. From their peak areas, the molar ratio of isomaltose to 3-O- $\alpha$-D-glucosylisomaltose was estimated to be $5.6: 1$.

The formation of isomaltose and 3-O- $x$-D-glucosylisomaltose is consistent with the sequences of $(1 \rightarrow 3)$ - and $(1 \rightarrow 6)$-linked $D$-glucose residues in the dextran molecule. As reported previously ${ }^{16}$, in addition to the stepwise liberation of isomaltose from consecutive $\alpha$ - $(1 \rightarrow 6)$-linked $D$-glucose residues, the $G_{2}$-dextranase also hydrolyzes the tetrasaccharide having the sequence $\alpha$-D-Glc- $(1 \rightarrow 3)-\alpha-D-G l c-(1 \rightarrow 6)-\alpha-D-$ Glc- $(1 \rightarrow 6)-D-G l c$, to give glucose and the trisaccharide $\alpha-D-G l c-(1 \rightarrow 3)-\alpha-D-G l c-$ $(1 \rightarrow 6)-D-G l c$. If it is assumed that most D-glucose residues in dextran B-1355-S are joined by alternating $\alpha-(1 \rightarrow 3)$ and $\alpha-(1 \rightarrow 6)$ linkages, 3-O- $\alpha$-D-glucosylisomaltose could arise only from the presence of non-reducing, terminal $\alpha-(1 \rightarrow 3)$ linkages whereas isomaltose should be liberated from non-reducing, terminal $\alpha-(1 \rightarrow 6)$ linkages, and also from the terminal $\alpha-(1 \rightarrow 6)$ linkages created by the exo-action of $G_{2}-$ dextranase (see Fig. 3) on the dextran.

The liberation of isomaltose may proceed until the enzymic cleavage ceases near the branch points, probably leaving two or three glucose residues. Thus, the formation of isomaltose and 3-O- $\alpha$-D-glucosylisomaltose from alternate $(1 \rightarrow 3)$ - and $(1 \rightarrow 6)$-linked $\alpha$-D-glucose residues may be explained by assuming that the enzyme acts at the reducing ends of alternate 6-substituted $\alpha$-D-glucose groups, in a fashion similar to the hydrolysis of lichenan by cellulase from certain Streptomyces sp. ${ }^{20}$.

The high-molecular-weight fraction (F-1 in Fig. 1), which may be regarded as the limit-dextran, was subjected to methylation analysis, and the molar ratio of the methylated glucose components was compared with that from the native dextran (see Table I). The methylation data indicate that the limit-dextran has a highly branched structure, with an average repeating-unit of six glucose-residues, consisting of non-terminal $(1 \rightarrow 6)$ - (2.4 parts) and $(1 \rightarrow 3)$-linked $\alpha$-D-glucose residues (1.47 parts), together with a non-reducing terminal and branch-point residue. This result confirms that the alternating $(1 \rightarrow 6)$ - and $(1 \rightarrow 3)$-linked sugar residues are distributed uniformly in the entire dextran molecule.

On the basis of methylation and acetolysis data, Seymour et al. ${ }^{11}$ recently proposed two types of repeating-unit structures for dextran B-1355-S: one consisting 
of an $\alpha-(1 \rightarrow 5)$-linked backbone chain witb side chains of alternating $\alpha-(1 \rightarrow 3)$ and $(1 \rightarrow 6)$ linkages, and the second consisting of a backbone chain of alternating $\alpha-(1 \rightarrow 3)$ - and $(1 \rightarrow 6)$-linked units with single $\alpha$-D-glucosyl stubs attached by $(1 \rightarrow 6)$ bonds. Our experimental data do not support either of these structures.

The results of our methylation studies indicate that the ratio of internal $(1 \rightarrow 6)$ to $(1 \rightarrow 3)$ linkages is $1.3: 1$ in the native dextran, and $1.6: 1$ in the $G_{2}$-dextranase limit-dextran. These data suggest that both $(1 \rightarrow 6)$ and $(1 \rightarrow 3)$ linkages are uniformly distributed.

As regards the action of $\mathrm{G}_{2}$-dextranase on the 1355-S dextran, the methylation data strongly suggest that the enzyme probably does not attack the branch points or bypass them, but rather results in the stepwise hydrolysis of $(1 \rightarrow 6)$ linkages, which ceases at a certain distance from the branch points ${ }^{16}$. The action of the $\mathrm{G}_{2}$-dextranase results in the release of an average of four out of 10 glucose residues of the repeatingunit chain.

If the dextran molecule consists of a regular sequence of $(1 \rightarrow 6)$ - and $(1 \rightarrow 3)$ $\alpha$-D-glucosidic linkages, and if the dextran is assumed to possess a ramified, multiplybranched structure, our experimental findings lead to the structural model shown in Fig. 3.

This structure is supported by our recent studies on the isolation of oligosaccharides (by acetolysis and partial acid hydrolysis) from dextran 1355-S (Torii et al., unpublished). Among the acetolysis products isolated were tri- and tetra-

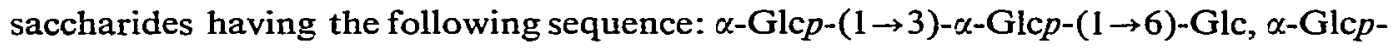

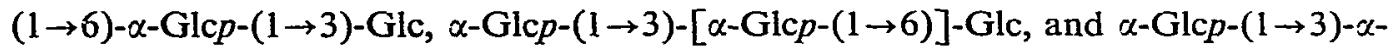
Glcp-(1 $\rightarrow 6)-\alpha-G l c p-(1 \rightarrow 3)-G i c$. A small proportion of nigerotriose was also obtained. Upon partial acid hydrolysis, the dextran gave, in addition to isomaltose, small amounts of isomaltotetraose. These oligosaccharides could have arisen from the sequences of alternate $\alpha-(1 \rightarrow 6)$ - and $\alpha$-(1 $1 \rightarrow 3)$-linked residues and the sequence of the residues around the branch points in the structure of dextran 1355-S (See Fig. 3).

In view of the physical properties of dextran-like $\alpha$-D-glucans that contain relatively high proportions of $\alpha-(1 \rightarrow 3)$-D-glucosidic linkages, it is apparent that the arrangements of $(1 \rightarrow 3)$ - and $(1 \rightarrow 6)$-linked sugar residues must play an important role in their water solubility. Thus, as already established, the water insolubility of OMZ 176 mutan may be attributed to the long stretches of consecutive $\alpha-(1 \rightarrow 3)$ linked D-glucose residues in the backbone chain, while short $\alpha-(1 \rightarrow 6)$-linked sidechains may be related to the adhesive properties ${ }^{7}$. The present experimental findings strongly suggest that the water solubility of dextran B-1355-S, which contains a high proportion of $\alpha-(1 \rightarrow 3)$ linkages, may be attributed to the alternating $(1 \rightarrow 3)$ and $(1 \rightarrow 6)$-D-glucosidic linkages, which may interfere with hydrogen bonding between adjacent chains. In this connection, it is noteworthy that the water-soluble glucan fraction synthesized by Streptococcus mutans OMZ 176 contains mainly $(1 \rightarrow 6)$ linked $\alpha$-D-glucose residues similar to those found in most dextrans (Misaki et al., unpublished results).

The interaction of concanavalin A with dextrans has been studied extensively, 


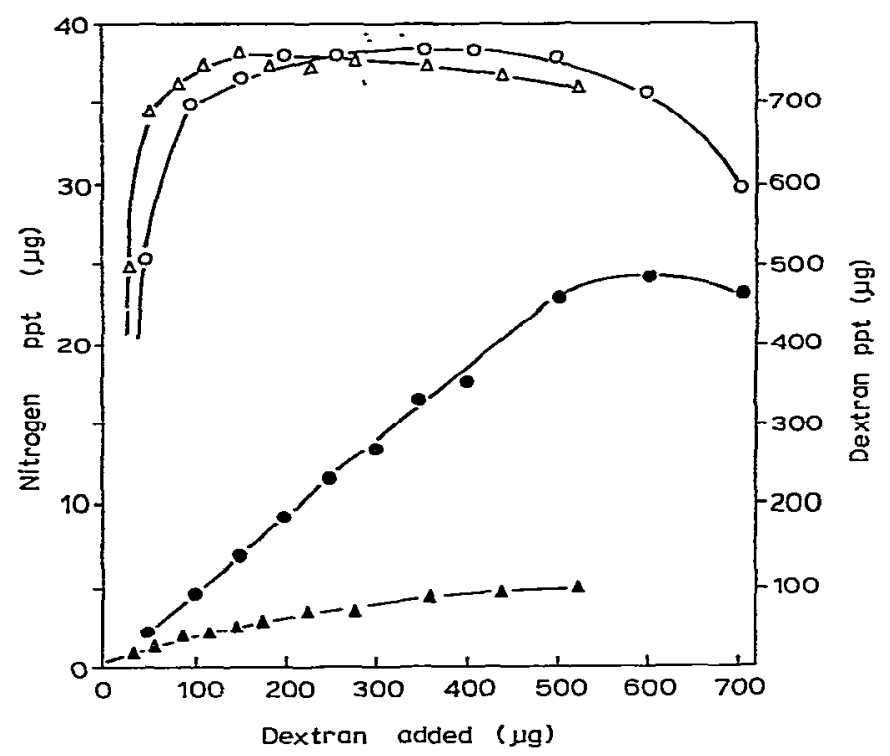

Fig. 4. Quantitative precipitation curves of dextran B-1355-S and $\mathbf{G}_{2}$-dextranase limit-dextrin with concanavalin $\mathrm{A}$. $-\mathrm{O}-$, protsin precipitated with native dextran: $-\triangle-$, protein precipitated with $\mathbf{G}_{2}$-dextranase limit-dextran; - - dextran in the precipitates of concanavalin A-dextran; - $\mathbf{A -}$, $\mathrm{G}_{2}$-dextranase limit-dextran in the precipitates of concanavalin $A-G_{2}$-dextranase limit-dextran.

and dextran B-1355-S has been used as the standard polysaccharide for the precipitation assay because of its great reactivity and the low solubility of the dextranlectin complex. Inasmuch as interaction with concanavalin A principally involves terminal $\alpha$-D-glucosyl groups of dextran molecules, the $G_{2}$-dextranase limit-dextran was compared with the native dextran in its reactivity with concanavalin A. As may be seen in Fig. 4, maximum precipitation of the protein with the limit-dextran occurs at one-half to one-third the amount of that of the native dextran.

This result may be due to the result of shortening of the chains by the action of $\mathrm{G}_{2}$-dextranase, so that the number of the non-reducing terminal-ends per unit weight increases. It is also noteworthy that the amount of the limit-dextran reacting with concanavalin A was found to be much less, approximately one-fourth that of the native dextran.

With regard to the immunochemical properties of dextran B-1355-S, the structural model proposed here can explain the previous observations that dextran B-1355-S reacts with human antidextran sera having specificity for both $(1 \rightarrow 3)$ and $(1 \rightarrow 6)-\alpha-D$-glucosidic linkages. When rabbit antiserum against $L$. mesenteroides B-1355 cells was fractionated on a column of Sephadex G-75, two immunochemically active fractions, one passing through, and the second binding to Sephadex, were obtained: the inhibition tests in both antibody-systems indicated that nigerose was a more potent inhibitor than isomaltose and other isomaltosaccharides ${ }^{23}$. In the precipitation system with the Sephadex-binding fraction, the inhibitory powers of 
isomaltosaccharides appeared to increase with their degree of polymerization, up to isomalto-tetraose or -pentaose, whereas in the system with the Sephadex non-binding fraction, all isomaltosaccharides showed the same inhibitory activity. The inhibitory activities of isomaltosaccharides, in the system with the Sephadex-binding fraction, may be related to the presence of small proportions of contiguous $(1 \rightarrow 6)$-linked $\alpha$-D-glucose residues in the dextran, as suggested by the detection of small amounts of isomalto-triose and -tetraose among the products of mild acid hydrolysis of the dextran. It should be pointed out, with regard to the complexity of the antigenic determinants of dextran B-1355-S, that among eight different oligosaccharides, inciuding a series of isomaltosaccharides and those containing bull $\alpha-(1 \rightarrow 6)$ and $\alpha-(1 \rightarrow 3)$ linkages, $6-O-\alpha-D$-glucosylnigerose was shown to be the best inhibitor in the precipitation system with the Sephadex-binding fraction; on the other hand, 3-O$\alpha$-D-glucosylisomaltose was the best inhibitor in the system with the fraction that passed through the Sephadex column (Torii et al., unpublished results). Further studies on the relationship between immunochemical specificities and the structure of dextran B-1335-S are in progress.

\section{EXPERIMENTAL}

Materials. - Dextran (soluble fraction) of Leuconostoc mesenteroides NRRL dextran B-1355-S, used in this study, was generously donated by $\mathrm{Dr}$. A. Jeanes, Northern Regional Research Laboratory, Peoria, IL, U.S.A. Exoisomalto- $\left(G_{2}\right)-$ dextranase ${ }^{16}$ and exo-( $\left.1 \rightarrow 6\right)-\alpha$-D-glucosidase ${ }^{18}$ were purified as reported previously. An endo-dextranase preparation from Penicillum sp. was purchased from Sigma Chem. Co. $(1 \rightarrow 3)-\alpha-D-G l u c a n$ hydrolase of Flavobacterium was prepared by the method reported in a previous paper ${ }^{15}$.

General methods. - Paper chromatography was performed on Whatman No. 1 or Toyoroshi No. 51A paper by the descending method using the following solvent systems (v/v): (A) 4:1:5 1-butanol-acetic acid-water, (B) 6:4:3 1-butanol-pyridinewater, and $(C)$ 6:1:3 1-propanol-ethanol-water. Preparative paper chromatography was performed with Whatman 3mm paper. Reducing sugars were detected by spraying the chromatograms with $p$-anisidine hydrochloride in water-saturated butanol, and alditols and glycosides of glucose were detected with Tollens' alkaline silver nitrate reagent. G.l.c. was conducted with a Shimazdu Gas Chromatograph Model CG-6AM equipped with a flame-ionization detector, using a glass column $(0.4 \mathrm{~cm}$ i.d.). Methylated sugars were converted into their corresponding alditol acetates, and separated at $180^{\circ}$ on a column packed with $3 \%$ of ECNSS-M on GasChrom Q. G.l.c.-m.s. was performed on alditol acetates with a Shimadzu-LKB gas chromatograph-mass spectrometer. For g.l.c. separation of the products from complete Smith-degradation, the hydrolysis products were treated with sodium borohydride and then acetylated. The products from the mild Smith-degradation were analyzed as their trimethylsilylated derivatives by g.l.c. on a column of OV-17, programmed from 80 to $320^{\circ}$ ( $6^{\circ}$ per min). 
Automated liquid-chromatography was performed with a Jeol liquid chromatograph Model JLC-3BC (Japan Electronic Optical Laboratory, Tokyo) using a column of anion-exchange resin (Jeol LCR-3). The oligosaccharides were separated ${ }^{19}$ by stepwise elution with $0.13 \mathrm{M}(\mathrm{pH} 7.5), 0.25 \mathrm{M}(\mathrm{pH} 9.0)$, and $0.35 \mathrm{M}(\mathrm{pH} \mathrm{9.6)}$ borate buffer, at $65^{\circ}$.

Total carbohydrate content was usually determined colorimetrically by the phenol-sulfuric acid method ${ }^{24}$.

Methylation analysis. - Polysaccharides were methylated according to the method of Hakomori ${ }^{12}$. Dry samples (20-30 mg each) of the dextran and its limitdextrin were dissolved in dimethyl sulfoxide $(2 \mathrm{~mL})$ by ultrasonication $(20 \mathrm{KHz}$, $5 \mathrm{~min})$ at $25^{\circ}$ in a nitrogen atmosphere, and methylated by treatment with methylsulfinyl carbanion $(1 \mathrm{~mL})$, followed by methyl iodide $(2 \mathrm{~mL})$. The mixture was dialyzed, concentrated to low volume, and the methylated product extracted with chloroform. If methylation was incomplete, as judged by i.r. spectroscopy, the methylation procedure was repeated. The methylated polysaccharide $(15 \mathrm{mg})$ was hydrolyzed by heating it with formic acid $(0.5 \mathrm{~mL})$ in a sealed tube for $10 \mathrm{~h}$ at $100^{\circ}$, the formic acid was distilled off, and the residue was heated further with $0.5 \mathrm{M}$ sulfuric acid $(0.3 \mathrm{~mL})$ for $3 \mathrm{~h}$ at $100^{\circ}$. The hydrolyzate was made neutral with barium carbonate, filtered, and the filtrate cvaporated to a syrup. A portion of the hydrolyzate was examined by paper chromatography (solvent $A$ ), and the remainder was reduced with sodium borohydride, converted into the acetates by heating with 1:1 pyridine-acetic anhydride $(0.2 \mathrm{~mL})$ for $1 \mathrm{~h}$ at $100^{\circ}$, and analyzed by g.l.c.

Periodate oxidation and Smith degradation of dextran. - The native dextran $(1.5 \mathrm{~g})$ was oxidized with $0.05 \mathrm{M}$ sodium periodate $(1500 \mathrm{~mL})$ at $5^{\circ}$ in the dark; at suitable intervals, the periodate consumption and formic acid production were assayed. After oxidation for 7 days, the excess of periodate was decomposed by the addition of ethylene glycol, and the solution was dialyzed for $24 \mathrm{~h}$ at $5^{\circ}$. To the nondialyzable solution was added sodium borohydride $(500 \mathrm{mg}$ in $100 \mathrm{~mL}$ of water), and the mixture kept overnight. Excess of borohydride was decomposed by careful addition of acetic acid to $\mathrm{pH}$ 7.2. The solution containing dextran-polyalcohol was dialyzed for 2 days at $5^{\circ}$, and evaporated (below $37^{\circ}$ ) to low volume, from which the dextran-polyalcohol was precipitated by pouring into acetone. The dextran-polyalcohol was collected by centrifugation, washed successively with abs. alcohol and ether, and dried in vacuo (yield, $1.07 \mathrm{~g}$ ).

Methylation of the foregoing dextran-polyalcohol $(50 \mathrm{mg})$ was achieved by two Hakomori methylations. The methylated dextran-polyalcohol was hydrolyzed by heating with $2 \mathrm{M}$ trifluoroacetic acid for $12 \mathrm{~h}$ at $100^{\circ}$. After repeated evaporations in the presence of water to remove the acid, the methylated sugar components were analyzed by g.l.c. as their alditol acetates, yielding 2,4,6-tri- and 2,4-di- $O$-methyl-Dglucose, together with a trace of 2,3,4,6-tetra- $O$-methyl-D-glucose (see Table I).

The dextran-polyalcohol $(10 \mathrm{mg})$ was hydrolyzed with $\mathrm{M}$ sulfuric acid $(0.3 \mathrm{~mL})$ for $2 \mathrm{~h}$ at $100^{\circ}$, as stated previously ${ }^{25}$. The hydrolyzate was made neutral with barium carbonate, and the neutral solution was treated with sodium borohydride in order 
to convert glucose in the hydrolyzate into glucitol. After removing inorganic salts, the reduced product was acetylated, and analyzed by g.l.c. on a column of $3 \%$ ECNSS-M at $65-190^{\circ}$, programmed at the rate of $5^{\circ}$ per min. The hydrolysis products were identified as glycerol and glucose, in the molar ratio of 1.0:0.83.

In another experiment, the dextran-polyalcohol (50 $\mathrm{mg})$ was subjected to mild acid hydrolysis with $0.05 \mathrm{M}$ sulfuric acid $(2 \mathrm{~mL})$ for $18 \mathrm{~h}$ at $25^{\circ}$. The hydrolyzate was made neutral with barium carbonate, and the products were examined by paper chromatography (solvent $B$ ) which revealed the presence of glycerol and $1-O-\alpha-\mathrm{D}-$ glucosylglycerol $\left(R_{\mathrm{Glc}} \mathbf{0 . 8 9}\right)$. These compounds were separated on a filter-paper sheet with solvent $B$, and each component was extracted with water. The glucosylglycerol showed $[\alpha]_{D}+134.8^{\circ}$ ( $c$ 1.4, water), and gave, on acid hydrolysis, equal proportions of glycerol and glucose. The glycerol and 1-O- $\alpha-\mathrm{D}-$ glucosylglycerol were extracted from the paper and determined colorimetrically by the chromotropic acid 26 and phenol-sulfuric acid methods, respectively; their molar ratio was $1: 1.45$. These components were also identified by g.l.c. on a column of OV-17 after conversion into their trimethylsilylated derivatives.

Degradation of the dextran by exo- $G_{2}$-dextranase and characterization of the products. - The dextran (100 $\mathrm{mg}$ ) was incubated with exo- $\mathrm{G}_{2}$-dextranase (45 units) at $\mathrm{pH} 4.5$ and $37^{\circ}$ in a total volume of $14 \mathrm{~mL}(0.05 \mathrm{M}$ acetate buffer $)$ in the presence of a drop of toluene. At suitable intervals, the increase in reducing groups was measured by the method of Nelson and Somogyi (after 2 days, 32.37\%; 4 days, $37.8 \%$; and 5 days, $38.2 \%$ ). After 5 days, the enzyme was inactivated by heating for $3 \mathrm{~min}$ at $85^{\circ}$, and the enzyme digest was subjected to gel-filtration on a column $(1.5 \times 60 \mathrm{~cm})$ of Sephadex G-25, which afforded two carbohydrate-components; Fraction 1 (65 mg) emerging in the void volume, and Fraction 2 (32 $\mathrm{mg})$ corresponding to oligosaccharides.

Paper chromatography (solvent $C$ ) showed that Fraction 2 consisted of a mixture of isomaltose $\left(R_{\mathrm{Glc}} 0.78\right)$ and a trisaccharide $\left(R_{\mathrm{Glc}} 0.66\right)$. They were separated from each other by preparative paper chromatography with Whatman 3m paper, giving isomaltose $(23 \mathrm{mg})$ and a trisaccharide $(8 \mathrm{mg})$, which showed $[\alpha]_{D}+130^{\circ}$ (c 1.0, water). A portion ( $3 \mathrm{mg}$ ) of the trisaccharide was dissolved in dimethyl sulfoxide $(0.5 \mathrm{~mL})$ and methylated by reaction with methylsulfinyl carbanion $(0.5 \mathrm{~mL})$ followed by methyl iodide $(1 \mathrm{~mL})$. The methylated trisaccharide in the mixture was extracted with chloroform. After hydrolysis with $2 \mathrm{M}$ trifluoroacetic acid for $8 \mathrm{~h}$ at $100^{\circ}$, the methylated sugar-fragments from the methylated trisaccharide were examined by g.l.c. as their alditol acetates. The presence of 2,3,4,6-tetra-, 2,3,4-tri-, and 2,4,6-tri- $O$-methyl-D-glucose, in the molar ratios of 1.0:1.0:0.95, was established. A portion $(1 \mathrm{mg})$ of the trisaccharide was incubated with yeast $\alpha$-D-glucosidase at pH 5.0 for $15 \mathrm{~h}$. Isolation by paper chromatography revealed the release of glucose and isomaltose. Identification and quantitation of isomaltose and trisaccharide were performed by means of automated liquid-chromatography (see Fig. 2).

The degraded dextran (Fraction $1,20 \mathrm{mg}$ ) was methylated by the method of Hakomori, and the methylated product was hydrolyzed with acid, and analyzed by 
g.l.c. as the alditol acetates, giving 2,3,4,6-, tetra- $O$-, and 2,3,4- and 2,4,6-tri- $O$-methylD-glucose (see Table I).

Quantitative precipitation of the native dextran and its $G_{2}$-dextranase limit dextran with concanavalin $A$. - Concanavalin A (Calbiochem, $300 \mu \mathrm{g}$ ) was precipitated with increasing amounts of dextran B-1355-S, and the $\mathrm{G}_{2}$-dextranase limitdextran (Fraction 1) in saline $(1 \mathrm{~mL}$ ) containing $0.018 \mathrm{M}$ phosphate buffer, $\mathrm{pH} 7.2$, according to the method of So and Goldstein ${ }^{27}$. After washing with saline, the precipitates were dissolved in $0.5 \mathrm{M}$ acetic acid $(3.0 \mathrm{~mL})$ and appropriate aliquots were subjected to nitrogen determination according to Schiffman et al. ${ }^{28}$. Aliquots were also analyzed for polysaccharide by the phenol-sulfuric acid method.

\section{ACKNOWLEDGMENTS}

We are grateful to Dr. A. Jeanes for providing a sample of dextran B-1355-S. A part of this work was supported by N.I.H. Grant AM 10171.

\section{REFERENCES}

1 R. L. Sidebotham, Adv. Carbohydr. Chem. Biochem., 30 (1975) 371-444.

2 B. Lindererg And S. SVensSON, Acta Chem. Scand., 22 (1950) 1907-1912.

3 H. MiYait, A. Misaki, AND M. Tori, Carbohydr. Res., 31 (1973) 277-287.

4 K. Sakakibara, M. Torit, B. P. Alberto, AND A. Misakr, Biochem. Biophys. Res. Commun., 73 (1976) 741-750.

5 E. J. Bourne, R. L. Sidebotham, ANd H. Weigel, Carbohydr. Res., 22 (1972) 13-22.

6 M. Kobayashi, K. Shishdoo, T. Kikuchi, and K. Matsuda, Agric. Biol. Chem., 37 (1973) 2763-2769.

7 S. Ebisu, A. Misaki, K. Kato, AND S. Kotani, Carbohydr. Res., 38 (1974) 374-381.

8 A. Jeanes, W. C. Haynes, C. A. Wilham, J. C. Rankin, E. H. Melvin, M. J. Austin, J. E. Cluskey, B. E. Fisher, H. M. TsuchiYA, AND C. E. Rist, J. Am. Chem. Soc., 76 (1954) 5041-5052.

9 W. J. Sloan, B. H. AleXander, R. L. Lohmar, Jr., I. A. Wolff, and C. E. Rist, J. Am. Chem. Soc., 76 (1954) 4429-4434.

10 I. J. Goldstein and W. J. Whelan, J. Chem. Soc., (1962) 170-175.

11 F. R. Seymour, M. E. Slodki, R. D. Plattner, and A. Jeanes, Carbohydr. Res., 53 (1977) 153-166.

12 S. HAKOMOR, J. Biochem. (Tokyo), 55 (1964) 205-208.

13 M. Abdel-Akher, J. K. Hammton, R. Montgomery, and F. Smith, J. Am. Chem. Soc., 74 (1952) 4970-4971.

14 I. J. Goldstein, G. W. Hay, B. A. Lewis, and F. Smith, Abstr. Pap. Am. Chem. Soc. Meet., 135 (1959) 3D.

15 S. Ebisu, K. Kaito, S. Kutani, and A. Misaki, J. Bacteriol., 124 (1975) 1487-1501.

16 T. Sawai, K. Toriyama, and K. Yano, J. Biochem. (Tokyo), 75 (1974) 105-112.

17 M. Toril, K. Sakakibara, A. MiSakr, and T. Sawai, Biochem. Biophys. Res. Commun., 70 (1976) 459-464.

18 T. Sawat, T. Yamaki, and T. Ohya, Agric. Biol. Chem., 40 (1976) 1293-1299.

19 M. TORI AND K. Sakakibara, J. Chromatogr., 96 (1974) 255-257.

20 A. S. Perlin and S. Suzuki, Can. J. Chem., 40 (1962) 50-58.

21 P. Z. Allen and E. A. Kabat, J. Am. Chem. Soc., 78 (1956) 1890-1894.

22 P. Z. Allen AND E. A. KaBAT, J. Immunol., 81 (1959) $4382-4386$.

23 B. P. Aleerto, M. TORI, AND A. TANaka, Proc. Jpr. Soc. Immunol., 7 (1977) 205-206. 
24 M. Dubois, K. A. Gllles, J. K. Hamilton, P. A. Rebers, and F. Smith, Anal. Chem., 28 (1956) 350-356.

25 A. Misaki and S. Yukawa, Agric. Biol. Chem., 32 (1968) 432-440.

26 D. J. Hanahan and I. N. Olley, J. Biol. Chem., 231 (1958) 813-823.

27 L. L. So AND I. J. Goldstein, J. Biol. Chem., 249 (1967) 1617-1622.

28 G. Schiffman, E. A. Kabat, AND W. Thompson, Biochemistry, 3 (1964) 113-120. 